\title{
Hyperinflation et mouvements de la rente dans les campagnes d'île-de-France
}

Fortunes et infortunes d'une bourgeoise rurale

Hyperinflation and movements in rent in the countryside of the Ile-de-France.

Fortunes and misfortunes of a rural Bourgeoisie

\section{Laurent Herment}

\section{(2) OpenEdition}

Journals

Édition électronique

URL : https://journals.openedition.org/ahrf/12943

DOI : $10.4000 /$ ahrf.12943

ISSN : 1952-403X

Éditeur :

Armand Colin, Société des études robespierristes

Édition imprimée

Date de publication : 1 décembre 2013

Pagination : 129-155

ISBN : 978290832789

ISSN : 0003-4436

Référence électronique

Laurent Herment, « Hyperinflation et mouvements de la rente dans les campagnes d'île-de-France », Annales historiques de la Révolution française [En ligne], 374 | octobre-décembre 2013, mis en ligne le 01 décembre 2016, consulté le 01 juillet 2021. URL : http://journals.openedition.org/ahrf/12943; DOI : https://doi.org/10.4000/ahrf.12943 


\title{
HYPERINFLATION ET MOUVEMENTS DE LA RENTE DANS LES CAMPAGNES D'ÎLE-DE-FRANCE. FORTUNES ET INFORTUNES D'UNE BOURGEOISE RURALE $E^{1}$
}

\author{
Laurent HERMENT
}

\begin{abstract}
II n'existe que très peu d'études qui concernent spécifiquement la vague de remboursements de créances au sein du monde rural durant la période d'hyperinflation de l'an III. Ce phénomène est souvent analysé au prisme de la relation de domination que les villes exercent sur les campagnes à la fin de l'Ancien Régime. Pourtant, au-delà de ce rapport de domination, la masse de créances remboursées durant l'an III laisse transparaître, comme à Milly-la-Forêt, chef-lieu de canton de Seine-et-Oise, une multitude de relations de crédit internes au monde rural. Ce travail, qui retrace la déconfiture des rentiers bourgeois de la région de Milly, permet aussi d'illustrer et de caractériser la multiplicité des relations de crédit internes au monde rural et les comportements différentiels des paysans face à l'inflation.
\end{abstract}

Mots-clés : Économie, Révolution française, inflation, campagnes, Île-de-France

(1) Cet article est issu d'une communication présentée dans le cadre du workshop organisé à Gérone les 27-29 septembre 2012 par l'université de Gérone et le GDRI CRICEC : Becoming richer or poorer through debt. Private credit and social change in the countryside. 
Ce n'est que depuis une vingtaine d'années, depuis les célébrations du Bicentenaire de la Révolution, qu'en matière économique, l'historiographie du monde rural de la période révolutionnaire s'est renouvelée ${ }^{2}$. En ce qui concerne le phénomène de désendettement dans les campagnes durant la Révolution, il existe très peu d'études antérieures aux travaux de Gilles Postel-Vinay ${ }^{3}$. De ce point de vue, aujourd'hui encore, les paysans du Bassin parisien sont les parents pauvres de l'historiographie. Longtemps, leur situation économique durant la période révolutionnaire a été examinée au prisme des événements politiques parisiens. Nous songeons tout particulièrement aux travaux de Richard $\mathrm{Cobb}^{4}$ mais aussi à ceux de Serge Bianchi $^{5}$ ou de Jacques Dupâquier ${ }^{6}$. L'effet de l'inflation révolutionnaire, phénomène éminemment politique comme l'ont montré Philip T. Hoffman, Gilles Postel-Vinay et Jean-Laurent Rosenthal ${ }^{7}$, a plus préoccupé les économistes mais il n'existe pas, à notre connaissance, de travaux portant spécifiquement sur le mouvement de désendettement rural en région parisienne. Même en dehors de l'orbite parisienne, les historiens qui se sont intéressés au mouvement de remboursement des rentes ont privilégié les relations villes-campagnes. Ce tropisme urbain est compréhensible puisque les études notariales sont souvent situées dans les villes et que, jusqu'à la fin de l'Ancien Régime, les villes dominent les campagnes. C'est le cas à Milly-la-Forêt comme dans les autres régions. Pourtant, la lecture

(2) Voir à ce sujet La Révolution française et le monde rural, Paris, Éditions du CTHS, 1989. Voir aussi Gilles POSTEL-VINAY, « À la recherche de la révolution économique dans les campagnes », Revue économique, vol. XL, 1989, p. 1015-1045.

(3) Gilles POSTEL-VINAY, La terre et l'argent. L'agriculture et le crédit en France du XVIII siècle au début du XX $X^{e}$ siècle, Paris, Albin-Michel, 1998, p. 136 et $s q$. Pour des travaux antérieurs voir Pierre MASSÉ, "À travers un dépôt de minutes notariales », AHRF, $25^{\mathrm{e}}$ année, octobre-décembre 1953, p. 297-315 et Michel Vovelle, Ville et campagne au $18^{e}$ siècle (Chartres et la Beauce), Paris, Éditions sociales, 1980, p. 136-166.

(4) Richard COBB, « L'armée révolutionnaire dans le District de Pontoise (brumaire-germinal an 2) », AHRF, Tome vingt-deuxième, 1950, p. 194-220; «Les disettes de l'an II et de l'an III dans le district de Mantes et la vallée de la basse Seine », Paris et Île-de-France, Tome III, 1951, p. 227-251 et « Le complot militaire de ventôse an II. Note sur les rapports entre Versailles et Paris au temps de la terreur (mars-avril 1794) », Paris et Île-de-France, Tome VII, 1955, p., 221-250.

(5) Serge BIANCHI, «Les femmes dans les troubles religieux et de subsistances dans le sud de l'Île-de-France ", dans Les femmes et la Révolution française. Modes d'action et d'expression. Nouveaux droits - Nouveaux devoirs, Actes du colloque international 12-13-14 avril 1989, Université de Toulouse-Le Mirail, Marie-France BrIVE (éd.), Toulouse, Presses universitaires du Mirail, 1989 ; « Les journées de la faim de l'an III en milieu rural dans le sud de l'Île-de-France », Paris et Île-de-France, 1990, p. 237-242 et La Révolution et la première République au village, Paris, Éditions du CTHS, Paris, 2003.

(6) Jacques DUPÂQUIER, «La résistance des paysans du Vexin Français à la Révolution (1793-1794) », Paris et Île-de-France, Paris, 1990, p. 305-317.

(7) Philip T. HoffMan, Gilles Postel-VInAY et Jean-Laurent Rosenthal, Des marchés sans prix. Une économie politique du crédit à Paris 1660-1870, Paris, Éditions EHESS, 2001. 
des registres des actes civils publics, dressés par l'administration durant l'an III dans le bureau de Milly-la-Forêt, permet de prendre conscience que l'étude de la relation villes-campagnes n'épuise pas la question du remboursement des rentes durant la période d'hyperinflation. Par ailleurs, la source ne mentionne pas uniquement des remboursements de rentes mais aussi de nombreux actes internes au monde rural, qui sont révélateurs à la fois de la diversité des relations de crédit et des attitudes de la paysannerie face à l'hyperinflation de l'an III. C'est en partant d'une étude centrée sur la personne d'Anne Élisabeth Lambert, propriétaire à Milly-la-Forêt, que nous envisagerons ces problèmes.

Milly-la-Forêt est une petite ville située dans le département de Seine-et-Oise à 50 kilomètres au sud-est de Paris (carte en annexe I). En l'an III, le bureau comprend treize communes. Trois communes sont situées dans les marges de la Beauce d'Étampes. Les autres communes sont situées dans le Gâtinais français. Les économies des deux régions sont assez différentes. En Beauce, où la grande propriété et la grande culture occupent une place prédominante, les terroirs sont presque entièrement dédiés à la culture des céréales. Dans le Gâtinais, les petites et moyennes exploitations sont très nombreuses. L'économie de cette région est plus diversifiée. Si la culture des céréales n'est pas négligée, la vigne, l'élevage et le jardinage constituent des activités essentielles pour de nombreux exploitants. Les phénomènes évoqués dans le cadre de cette communication concernent plus particulièrement la partie gâtinaise du bureau dans laquelle les propriétés bourgeoises et paysannes sont très importantes.

Les registres des actes civils publics tenus par l'administration de l'enregistrement pendant l'an III au bureau de Milly-la-Forêt constitueront la source quasi-exclusive de ce travail ${ }^{8}$. Deux types d'actes sont consignés dans les registres de l'administration fiscale : les actes dressés par les notaires (baux de terre, ventes, obligations, constitutions de rente, créations de rente foncière, quittances, compromis, transactions, contrats de mariages, inventaires, etc.) et les actes sous seing privé que les parties désiraient faire enregistrer afin de disposer d'une preuve de leur existence. En ce qui concerne les notaires, ils étaient tenus de procéder à l'enregistrement des actes qui étaient passés devant eux dans un délai de quinze jours ${ }^{9}$.

(8) AD Essonne, cotes 3 Q10 / 3 et 3 Q10 / 4. Pour une présentation succincte de cette source et de la bibliographie qui y est consacrée voir Gérard BÉAUR, « Révolution et transmission de la propriété : le marché foncier ordinaire (Lizy-sur-Ourcq et Bar-sur-Seine entre 1780 et 1810), La Révolution française et le monde rural, op. cit., p. 271-286.

(9) L'enregistrement avait lieu au bureau dans le ressort duquel était située leur étude. 
Les délais d'enregistrement des actes sous seing privé sont nettement plus aléatoires.

Durant l'an III, de très nombreuses quittances sont enregistrées. Ce n'est pas le rythme des remboursements qui focalisera l'attention dans le cadre de ce travail. Il est le même qu'ailleurs (graphique annexe II). Après une première poussée de fièvre au début de l'hiver 1794-1795, les remboursements se multiplient au printemps 1795. En thermidor, le nombre et le volume des remboursements s'effondrent brutalement pour deux raisons : la source et la loi. Il manque plusieurs folios dans le registre 3 Q 10/4 des archives départementales de l'Essonne. Ils correspondent aux 15 derniers jours de thermidor et au mois de fructidor. Mais en thermidor an III, la cause est entendue puisque la loi $\mathrm{n}^{\circ} 966$ du 25 messidor an III prévoit, en son article 2 , que « les remboursements de toutes les rentes créées avant le premier janvier 1792, quelle que soit leur nature et la cause dont elles procèdent, sont provisoirement suspendus ${ }^{10}$.

Le registre contient pour la période considérée 1165 quittances de rentes foncières ou constituées pour un montant de près de 350000 livres. 652 enregistrements mentionnent les dates de création des rentes ou, le cas échéant, les dates de renouvellement ou de transport des titres. Ainsi, il est possible de jeter un regard rétrospectif sur le mouvement de création des rentes durant le XVIII ${ }^{\mathrm{e}}$ siècle. Par ailleurs, en principe, les enregistrements nomment, domicilient et qualifient les parties aux actes. Il est donc possible de mesurer les pertes des bourgeoisies urbaines et les gains des communautés rurales essentiellement composées de manouvriers, de vignerons, de cultivateurs, de journaliers, etc. La source offre un autre parti. Durant cette période, en raison de l'inflation galopante, de nombreux individus prirent la précaution d'enregistrer diverses opérations économiques qui en d'autres circonstances n'auraient pas laissé de trace. Il est donc possible de saisir au plus près certaines des logiques qui structurent le tissu économique, à la fois dans ce qu'il a de déformé, compte tenu de l'inflation, mais aussi, et peut-être surtout, dans ce qu'il a de quotidien. Ainsi, la source formule un discours qui se conjugue au passé et un autre discours qui se conjugue au présent. Elle permet, en outre, de saisir une partie des choix en matière de crédit et une partie des comportements économiques des différentes classes de la société rurale : bourgeois (ou rentiers), nobles,

(10) Sur la protection des créanciers durant la période de dégringolade de l'assignat voir François CROUZET, La grande Inflation. La monnaie en France de Louis XIV à Napoléon, Paris, Fayard, 1993, p. 392 et $s q$. 
cultivateurs et laboureurs, plèbe rurale (journalier, manouvrier, vigneron), etc.

Dans la première partie, à partir de l'examen de la situation d'Anne Élisabeth Lambert, nous tenterons de montrer qu'il existe plusieurs réseaux de création de rentes. La seconde partie de ce travail sera l'occasion de s'interroger sur la sédimentation progressive au cours du XVIII ${ }^{\mathrm{e}}$ siècle d'un volume de rentes de diverses natures d'une part, et, d'autre part, sur le mouvement du crédit durant l'an III. Dans la troisième partie, on s'intéressera aux autres opérations mentionnées par la source. Il s'agira d'examiner les attitudes de la population rurale face aux occasions et aux dangers que présentait la dévalorisation accélérée de l'assignat, ce qui nous permettra de saisir, en creux, les multiples relations de crédit interne au monde rural.

\section{Taxinomie de la gente rentière}

\section{Une espèce sociale à Milly-la-Forêt : le rentier d'Ancien Régime}

Nous ne savons pas grand-chose des débuts dans la vie d'Anne Élisabeth Lambert, fille d'Adrien Lambert et d'Anne Élisabeth Marchand, si ce n'est qu'elle est née à Versailles, vers 1734, et qu'à une date indéterminée elle s'est mariée avec Louis Ambroise Chauvin. Nous ignorons aussi à quelle date elle s'installe à Milly-la-Forêt où elle décède le 2 février 1803 (13 pluviôse an XI), à l'âge de 69 ans, veuve d'Ambroise Chauvin ${ }^{11}$. Nous savons, par contre, que la famille Lambert possède environ 100 hectares dans dix communes lors de l'établissement du cadastre des communes du canton de Milly-la-Forêt en 1811-1817. Il s'agit donc d'une famille bourgeoise implantée à Versailles, Paris et Milly-la-Forêt, qui fait jeu égal avec la petite noblesse du Gâtinais.

Au cours du XVIII siècle, la famille Lambert ne se contente pas d'accumuler des terres. À l'orée de la Révolution, Anne Élisabeth dispose de très nombreuses rentes foncières ou constituées qui grèvent les patrimoines de la paysannerie locale depuis parfois plus d'un siècle. Cette stratégie de longue haleine, qui ne se réduit pas à une stratégie de conquête du sol, se heurte durant la Révolution à une vague inouïe de remboursements. En moins d'un an, durant l'an III, Anne Élisabeth reçoit plus de 70

(11) Louis Ambroise Chauvin est qualifié dans l'acte de mariage de sa fille de procureur au parlement. Il décède à Paris à une date indéterminée, État civil de Versailles, AD Yvelines, Mariage de Marie Élisabeth Chauvin et de Paul Hyacinthe Sainglant, Registre des mariages 1812, page 41. 
remboursements de rentes pour un montant supérieur à 20000 livres, soit la valeur de 40 hectares de terre environ ${ }^{12}$.

Il convient de rappeler ici la différence entre les deux types de rentes. Comme l'indique Geneviève Massa-Gille la rente foncière (ou de bail d'héritage) «était une redevance fixe en argent ou en denrée, créée et réservée sur un immeuble, fonds de terre, maison, propriété urbaine ou rurale, lors de son aliénation. Elle transférait au preneur [le débirentier] une portion de la propriété du fonds $»^{13}$. La rente constituée, quant à elle, correspondait à un prêt d'argent par le crédirentier au débirentier contre une rente annuelle. La rente foncière était, en principe, perpétuelle (non rachetable). Il s'agissait, ni plus ni moins, d'un acte par lequel la propriété d'un fond était transférée au débirentier à charge pour lui de payer annuellement une rente. La rente constituée, qui n'avait pas du tout le même caractère, puisqu'il s'agissait d'un prêt d'argent ${ }^{14}$, était en principe rachetable à la volonté du débiteur. Mais, le temps aidant, ces rentes constituées se transformaient en rente perpétuelle. Ce n'est qu'en période d'inflation que le débirentier avait intérêt à rembourser le capital de la rente ${ }^{15}$.

La déclaration de succession d'Anne Élisabeth nous apprend peu de chose sur sa biographie. On devine qu'elle et sa fille sont pieuses. C'est Étienne Coissard, pensionnaire ecclésiastique, domicilié chez la défunte, qui procède à l'enregistrement de la fortune fiscale de la défunte. Sur le plan financier, la succession mobilière d'Anne Élisabeth s'élève à 20000 francs. En l'absence d'inventaire, nous n'en saurons pas plus. La succession immobilière fait mention d'un patrimoine de 21885 francs $^{16}$. Donc, à l'issue de la Révolution, loin d'être totalement ruinée, Anne Élisabeth dispose encore d'un beau patrimoine foncier et immobilier. Elle habite au 185 rue des pelletiers, dans une maison estimée 5000 francs. Elle loue à la veuve Salar une autre maison sise dans la même rue, moyennant 150 francs par an. Elle loue environ 19 hectares de terre et courtil et divers

(12) Dans le canton de Milly-la-Forêt (limite de l'an III), l'hyperinflation a sans doute entraîné, au profit de la petite paysannerie, un transfert de richesse plus important que la vente des biens nationaux. Voir à ce sujet Gilles PoSTEL-VINAY, La terre et l'argent..., op. cit., p. 144.

(13) Geneviève MASSA-GILLE, «Les rentes foncières sous le Consulat et l'Empire », Bibliothèque de l'école des chartes, 1975, tome 133, livraison 1, p. 59-91, p. 59.

(14) D'un point de vue formel, la rente constituée différait du prêt à intérêt en ce que le débirentier n'était débiteur que de la rente (de l'annuité) et non du principal.

(15) Il faut souligner qu'un bien ou la totalité du patrimoine du débirentier servait de garantie au paiement de ce type de rente.

(16) AD Essonne, cote 3Q10/266 (Table des successions) et 3Q/10/267 (Registre des mutations). L'enregistrement a lieu le 11 ventôse an XI. 
bâtiments à Milly-la-Forêt et Boigneville pour un montant annuel de 510 365 francs. Enfin, elle possède 9 hectares de bois à Milly-la-Forêt ${ }^{17}$. Bref, si l'inflation révolutionnaire a écorné cette petite fortune, elle n'a pas privé Anne Élisabeth et sa fille de toutes leurs ressources. Afin de mieux situer Anne Élisabeth, il convient de répondre à deux questions. Constitue-t-elle un cas d'espèce ou un cas parmi d'autres ? Qui sont les débiteurs qui se pressent en foule pour lui rembourser à très bon compte le capital des rentes que sa famille a accumulées?

Anne Élisabeth n'est pas la seule victime de l'hyperinflation de l'an III. Pierre de Villerval (le citoyen Villerval), propriétaire à Milly-laForêt, est délesté de 15000 livres. Gillet dit de la Rennomière, domicilié à Noisy, commune de Seine-et-Marne, limitrophe de Milly-la-Forêt, perd 3600 livres dans l'affaire. La famille Pasquet de Leyde abandonne plusieurs milliers de livres, etc. D'autres crédirentiers parisiens ne sont pas mieux lotis. Mauduisson et sa femme Le Camus perdent 900 livres. On rembourse presque 7000 livres à Alexandre Barreau-Dugué, etc. Plusieurs indices démontrent que ces victimes ne sont pas totalement ruinées. Pierre Villerval décède le 4 ventôse an XI. Il laisse à sa veuve une fortune meuble de 9000 francs composée du préciput auquel elle a droit et de l'usufruit du mobilier du défunt. Les collatéraux reçoivent 10970 francs de meubles. L'usufruit des immeubles qu'il laisse à sa veuve représente une valeur de 13994 francs. Les collatéraux de Pierre de Villerval reçoivent donc en nue-propriété des immeubles d'une valeur fiscale de 27988 francs $^{18}$. Comme Anne Élisabeth et Pierre de Villerval, les autres rentiers, dont l'existence est partagée entre Paris, Versailles ou Orléans et Milly-la-Forêt, parviennent à sauver l'essentiel de leur patrimoine.

La spécificité de cette catégorie de rentiers bourgeois (ou de petite noblesse) permet de préciser le rôle de trois autres catégories de crédirentiers. La première est constituée par deux familles nobles, l'une d'épée (Bizemont) et l'autre de robe (Nicolaÿ). La famille de Bizemont possède plus de mille hectares dans la région de Milly-la-Forêt. La famille Nicolaÿ possède 570 hectares à Courances et Dannemois. Les pertes de ces deux familles sont minimes. Huit rentes pour un montant de 5723 livres, arrérages compris, sont remboursées à la famille Bizemont, trois rentes, d'un montant de 1140 francs, sont remboursées à Philippine Léontine Potier de Novion,

(17) Il est possible qu'Anne Élisabeth possède des immeubles dans d'autres cantons du département ou à Paris. La structure de la source ne permet pas de le savoir.

(18) AD Essonne, cote 3Q10/266. 
veuve Nicolaÿ. Si, par ailleurs, une rente est remboursée à Dupré de SaintMaur, et deux autres à Daniel Victor de Trimond, aucune n'est remboursée aux familles d'Averton, Bellavenne, de Sainte-Croix, Du Lau d'Allemans, etc.

La petite bourgeoisie locale laisse aussi des plumes dans l'affaire. On se doute que, dans ces cas, la ponction est lourde. Ainsi, Anne Tousson ou Detousson, domiciliée à Milly-la-Forêt, veuve de Jean-Baptiste Dupré, laisse 1391 livres, arrérages compris, dans le remboursement de quatre rentes. Les paysans les plus riches sont aussi les victimes de la vague de remboursement. Jean Baptiste Chevalier, cultivateur, domicilié à Tousson, perd 2561 livres, dans le remboursement de dix rentes (arrérages compris) ${ }^{19}$. On citera, enfin, Jean-Jacques Delafosse, ancien notaire, domicilié à Maisse. Une foule de petits créanciers lui remboursent environ 10000 livres. Mais, comme les autres rentiers professionnels, il sauve l'essentiel de sa fortune foncière et immobilière ${ }^{20}$.

\section{Les rentes paysannes}

La petite paysannerie constitue la dernière catégorie de crédirentiers. Pour comprendre la spécificité de ce type de rente il faut citer la source. Le 18 germinal de l'an III, il est fait mention d'une :

«Quittance par Nicolas Hochard vigneron demeurant à Dannemois, Alexandre Thévenot demeurant aud lieu à cause de sa femme \& Jean Hochard demeurant à Cély [Seine-et-Marne]. À Alexandre Lépicier cultivateur demeurant à Videlles [Seine-et-Oise] \& Marie Jeanne Canet sa femme. De la somme de trois cent cinq livres. Savoir trois cents livres pour le remboursement de quinze livres de rente foncière et de bail d'héritage passé devant Audenet notaire à Dannemois le 20 février 1782 et cinq livres pour cinq mois d'arrérages d'icelle $»^{21}$.

(19) Par exception il est domicilié à Milly-la-Forêt une fois. Il est fait mention d'un autre Jean Baptiste Chevalier domicilié à Nemours. Nous ne savons pas s'il s'agit du même individu.

(20) On retrouve à travers ces personnages les profils des rentiers mis au jour à Chartres par Michel VoVELLE, Ville et campagnes..., p. 135-166.

(21) Par souci de lisibilité et en vue d'un traitement informatique nous n'avons pas retenu la graphie originale. En ce qui concerne cette quittance, le registre indique : «Une q q $^{\text {par Nicolas }}$ Hochard vigneron demt à Dannemois, Alexandre Thévenot demt aud. lieu acause de sa fe \& Jean Hochard demt à Cély. A Alexandre Lépicier cultivateur demt à Videlles \& Marie Jeanne Canet sa fe. De la somme de trois cent cinq livres. Savoir trois cents livres pour le remboursement de quinze livres de rente foncière et de bail d'héritage passé devant Audenet no ${ }^{\text {re }}$ à Dannemois le 20 février 1782 ; Et cinq livres pour cinq mois d'arrérages d'icelle » 
Cette quittance permet de mettre en exergue la particularité des créances internes au monde rural. Elle concerne trois crédirentiers. Il est clair, même si cela n'est pas explicitement précisé, que cette situation résulte d'un phénomène de transmission patrimoniale au sein de la famille Hochard. Elle nous met sur la voie d'un autre phénomène particulièrement important. Nicolas Hochard est apparu une première fois, le 4 germinal, en tant que débirentier cette fois :

«Une quittance par Jean Baptiste Bouvard vigneron à Dannemois, Barthélémy Bouchet, Louis Carré, Jacques Gaillard, Louis Poupinet et autres. À Nicolas Hochard vigneron demeurant à Dannemois. De deux cent cinquante-trois livres huit sols six deniers. Savoir deux cent cinquante livres pour le remboursement de douze livres dix sous de rente restante de dix-sept livres foncière et de bail d'héritage passé devant Charlot notaire à Dannemois le $297 \mathrm{bre} 1718$ et trois livres huit sous six deniers pour arrérages d'icelle »

Ici, au moins cinq crédirentiers différents sont spoliés par Nicolas Hochard $^{22}$. On peut montrer que ces remboursements constituent souvent un jeu à somme nulle. Les victimes de Nicolas Hochard vont à leur tour faire subir le même traitement à un ou plusieurs de leurs créanciers.

Il existe donc deux circuits d'endettement. Le premier met aux prises les bourgeois, petits et gros, avec les cultivateurs, les vignerons, les manouvriers du Gâtinais. Le second est interne à la paysannerie. Dans ce second circuit, les rentes dont se délestent les paysans constituent parfois le reliquat d'un partage, parfois la conséquence d'arrangements familiaux, souvent elles sont les fruits des mécanismes de circulation de la terre.

Dans une région de partage égalitaire, le remboursement en assignats des rentes foncières peut rompre l'égalité entre héritiers. Si l'on peut supposer que l'effacement d'une rente vieille d'un siècle, de cinquante ans, voire de vingt ans, ne laisse pas trop de regrets au crédirentier, il faut se souvenir que plus la rente est récente plus la perte est lourde. Pour les imprudents, qui continuent à pratiquer ce type d'exercice durant l'an II ou l'an III, quelques mois, voire quelques jours dans le pire des cas, peuvent séparer l'arrangement intrafamilial ou la vente d'un bien à un voisin et le versement du capital en monnaie de singe.

(22) L'éclatement des rentes sous l'effet des ventes et des partages successoraux concerne les débiteurs et les créanciers. Seuls les portefeuilles bourgeois échappent, en principe, à cette règle. 
Il convient de se demander qui, des bourgeois ou des paysans, a enregistré les plus grosses pertes. Globalement ce sont les bourgeois de Milly-la-Forêt, de Paris, d'Étampes, de Versailles, de Fontainebleau et des autres villes qui ont fait les frais de la vague de remboursements. Les pertes nettes des individus domiciliés à Milly-la-Forêt, s'élèvent à plus de 65000 livres. Avant la Révolution les bourgeois de Milly-la-Forêt sont situés au sommet de la chaîne d'endettement, il est donc logique, qu'en dernière analyse, ils soient les principales victimes de l'hyperinflation.

Les débiteurs d'Anne Élisabeth, comme ceux des autres bourgeois, sont, pour l'essentiel, des petits propriétaires, souvent qualifiés de cultivateurs, de manouvriers ou de vignerons ${ }^{23}$. Ils sont domiciliés dans les communes proches de Milly-la-Forêt. À une exception près, Courances, ces communautés rurales parviennent à se défaire de dettes qu'elles traînent parfois depuis plusieurs générations. Les habitants de Moigny remboursent près de 8468 livres aux individus domiciliés à Milly-la-Forêt, ceux de Maisse 6682 livres, ceux de Noisy [Seine-et-Marne] 5054 livres, etc. Outre Anne Élisabeth Lambert, Pierre de Villerval se fait étriller en compagnie d'Edme Verdier, de Françoise Lépinette, des membres des familles Charlot, Blondel et Pasquet, etc.

Gérard Béaur note qu'à "partir du moment où les campagnes étaient nettement débitrices des villes, il est clair que les ruraux sont les grands bénéficiaires de ce formidable transport, au moins dans un premier temps $»^{24}$. Les résultats que nous obtenons permettent de confirmer ce diagnostic. Mais il faut souligner qu'à Milly-la-Forêt la situation est particulièrement propice à ce type de constat. Il n'est pas certain que l'on retrouve le même phénomène dans une région où la rente n'est pas la forme dominante de placement bourgeois ou dans des régions dans lesquelles l'aristocratie et le clergé possèdent avant la Révolution une part très importante du sol, ce qui n'est pas le cas dans le Gâtinais, en dépit de l'importance des possessions de quelques familles nobles ${ }^{25}$.

(23) En ce qui concerne les débirentiers qui remboursent Anne Élisabeth, sur 44 qualifications, celle de cultivateur revient 16 fois, celle de vigneron 10 fois, celle de manouvrier 5 fois. On trouve par ailleurs parmi les débiteurs d'Anne Elisabeth, trois laboureurs, un berger, un notaire, un maçon, un marchand de chevaux, un marchand, un marchand boulanger, un tonnelier et un libraire domicilié à Paris.

(24) Gérard BÉAUR, « Révolution et redistribution des richesses dans les campagnes : mythes ou réalité ? », $A H R F, \mathrm{n}^{\circ} 352$, avril-juin 2008, p. 209-239.

(25) Pour une analyse de la répartition de la propriété au début du XIX ${ }^{\mathrm{e}}$ siècle dans la région de Milly-la-Forêt, voir Laurent Herment, Les fruits du partage. Petits paysans du Bassin Parisien au XIX $X^{e}$ siècle, PUR, Rennes, 2012, p. 33 et sq. 


\section{L'historique du stock de rente.}

\section{Montée de la rente au XVIIIe siècle dans le Gâtinais}

Il convient maintenant d'examiner la sédimentation de ce volume de rentes. Pierre Massé rappelle que dans le Châtelleraudais, au XVIII ${ }^{\mathrm{e}}$ siècle, « le flux de la rente, tant foncière que seigneuriale, coule à plein bord ${ }^{26}$. En ce qui concerne la rente foncière, c'est aussi le cas à Milly-la-Forêt ${ }^{27}$. Mais, d'une part, la rente foncière n'est pas le seul type de rente et, d'autre part, il faut tenir compte de l'existence éventuelle d'autres instruments de crédit (les obligations, les billets et les promesses de remboursements). On sait qu'à Paris, après l'épisode de Law, le stock d'obligations a augmenté et qu'à la fin du XVIII ${ }^{\mathrm{e}}$ siècle le flux de rentes constituées s'est tari ${ }^{28}$. Qu'en est-il à Milly-la-Forêt ?

Avant de répondre à cette question il est indispensable d'effectuer quelques mises au point méthodologiques ${ }^{29}$. En premier lieu, puisqu'on prétend retracer la montée de l'endettement, il convient de raisonner en termes de flux. Nous ne prétendons pas reconstituer le stock de dette au début de l'an III, même si l'on devine qu'il est très important. En second lieu, il faut connaître la date de création des rentes. Trois phénomènes polluent les données de ce point de vue. Le premier renvoie à la pratique des scribes qui se contentent de signaler l'existence de titres sans les mentionner explicitement. Ce sont plus de 100 quittances qui disparaissent de la base d'informations. Le second est lié à l'imprécision des déclarations. Il entraîne des pertes plus considérables encore. Le 21 nivôse, le scribe note : « une quittance par Jean Baptiste Chevalier cultivateur demeurant commune de Tousson. À François Deflandres, Jacques André, André Laure, Pierre Venet et autres ; pour le remboursement de vingt-trois livres dix sous de rente foncière. Sans énonciation de titres $»^{30}$.

Les titres nouvels et les transports de rente constituent le troisième problème $^{31}$. La date de création d'un titre nouvel, par exemple, ne nous

(26) Pierre MASSÉ, op. cit., p. 305.

(27) En l'an III, il n'est évidemment plus question de rente seigneuriale.

(28) Philip T. Hoffman, Gilles Postel-VinaY et Jean-Laurent Rosenthal, Des marchés sans prix..., op. cit., graphique p. 68

(29) Nous avons éliminé de ces calculs les 50 quittances qui concernent les remboursements de rentes dues aux institutions publiques.

(30) Les formules varient. Les scribes notent parfois : « sans autre désignation » ou, faisant allusion à une rente, ils inscrivent simplement " de la nature qu'elle est due... ».

(31) Un titre nouvel est un acte par lequel un débirentier reconnaît l'existence de la rente et s'oblige à continuer à la servir. Le transport consistait pour le crédirentier à transférer la rente au profit d'un tiers. 
renseigne en rien sur la date de création de l'acte originel. Il ne faut pas pour autant négliger ces quittances. Leur répartition dans le temps permet de s'assurer que la série dont on dispose fait sens. En effet, plus la rente est ancienne, plus la probabilité de disposer d'un titre nouvel ou d'un acte de transport est importante. La proportion de titre nouvel ou de transport devrait donc croître avec le temps. C'est effectivement ce qui se produit comme l'indique le graphique 1.

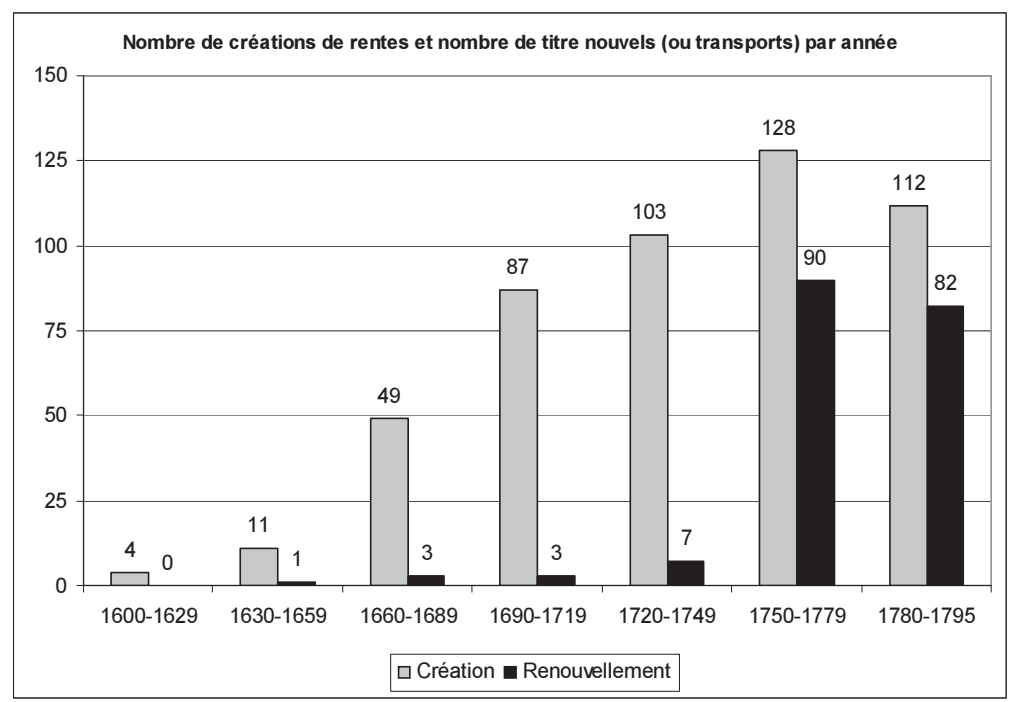

Graphique 1. Part des titres nouvels ou de transports dans les quittances.

Ce graphique fournit une autre information. La montée de l'endettement semble indéniable. Nos résultats ne confirment pas l'observation de Labrousse selon lequel « le flux de rente [...] tiédit le soir de l'Ancien Régime $»^{32}$. Cette montée est particulièrement nette à partir de $1720^{33}$. On pourra objecter qu'une partie des rentes les plus anciennes a été remboursée. Cette remarque ne vaut pas pour les rentes foncières qui sont en principe non rachetables. Or, si l'on isole cette catégorie de rentes, l'impression de croissance de l'endettement reste la même.

(32) Cité par Pierre MASSÉ, « À travers un dépôt... », op. cit., p. 395.

(33) Sauf à considérer que les renouvellements de rente des années 1750-1795 concernent exclusivement des rentes dont la création est antérieure à 1720, ce qui est assez peu probable sans être impossible, il faut admettre que la création de rente ne tiédit pas à la fin de l'Ancien Régime. 
Si l'on s'intéresse exclusivement aux actes pour lesquels on connaît la date de création originelle, on constate deux phénomènes. Le premier ne nous apprend pas grand-chose : le nombre de créations croît au fur et à mesure du siècle. Contrairement à ce qui se passe à Paris, le mouvement de créations ne se tarit pas durant la décennie 1780, même s'il est peutêtre moins important que durant les décennies 1730-1750. Le second phénomène est beaucoup plus intéressant. Il concerne le mouvement de création des rentes constituées. On s'attend à ce qu'une période se dégage de cette série : la période Law. C'est effectivement le cas comme l'indique le graphique 2. À Milly-la-Forêt, comme à Paris, les années 1719-1720 correspondent à un très fort mouvement de création de rente constituée, ce qui n'a rien d'étonnant compte tenu de la position géographique de Milly-la-Forêt ${ }^{34}$.

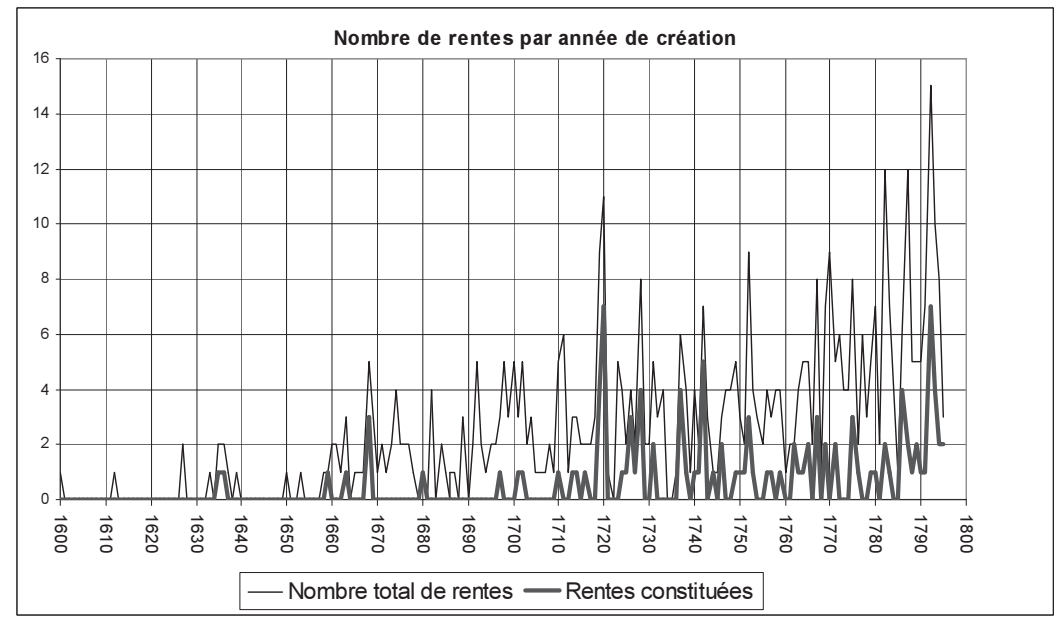

Graphique 2. Année de création des rentes remboursées au cours de l'an III.

\section{Le mouvement de création d'obligations et de rentes durant l'an III}

Si à la fin du XVIII e siècle les obligations constituent l'instrument de crédit privilégié à Paris, dans le Gâtinais elles représentent un instrument de crédit assez récent. Par ailleurs, les obligations sont des instruments de crédit à court ou moyen terme. En principe, elles sont remboursées dans un 
délai de quelques années. De ce point de vue, la vague de remboursements de l'an III ne conserve pas la mémoire des flux passés. Il est donc très difficile de mener une analyse rétrospective. Quant aux billets ou aux promesses ils se comptent sur les doigts d'une main. C'est le passage du passé au présent de la source qui révèle le phénomène le plus intéressant : il réside dans le mouvement de création d'obligations et de rentes constituées durant l'an III.

Si à Paris le mouvement de création de rentes constituées s'éteint à la fin de l'Ancien Régime, il n'en est rien à Milly-la-Forêt. En fait, loin d'abandonner cette pratique, les habitants de Milly-la-Forêt vont s'y livrer avec frénésie durant l'an III. Pour comprendre ce phénomène il faut évoquer les problèmes auxquels font face les emprunteurs et les prêteurs.

En période d'hyperinflation, la rente foncière représente le pire des placements. Reste aux prêteurs à choisir entre les obligations, les rentes constituées et les rentes viagères. Le travail de Philip T. Hoffman, Gilles Postel-Vinay et Jean-Laurent Rosenthal peut nous servir de guide. On sait que les obligations représentent pour les prêteurs un gage de relative sécurité puisqu'ils maîtrisent la date de remboursement. Les prêts de ce type se multiplient durant l'an III (voir graphique 3). Chaque enregistrement précise l'échéance : un an, dix-huit mois, deux ans, etc. Les plus audacieux jouent le court terme, les plus prudents tablent sur un retour à la normale dans un horizon plus lointain. Cette micro-série démontre que certains prêteurs adaptent très vite leurs pratiques aux circonstances. Pourtant, en dépit des risques que présentent les placements en rentes constituées, de nombreux prêteurs restent fidèles à ce type de placement.

Sur 60 rentes créées durant l'an III, 8 sont foncières, 48 sont constituées et 2 sont viagères ${ }^{35}$. Comment expliquer que les prêteurs acceptent de prendre le risque de prêter sous cette forme? Réglons tout de suite le sort des foncières. Sur les 8 rentes créées durant l'an III, six le sont entre septembre 1794 et janvier 1795. Après cela le mouvement de création s'épuise (graphique 3).

Comment expliquer l'abondance de rentes constituées? On peut voir dans ce phénomène la myopie des agents. Mais il faut alors renoncer à expliquer la croissance du nombre d'obligations par leur faculté d'adaptation. On est d'autant moins enclin à avancer cette explication que les prêteurs ne sont pas des oisons. 


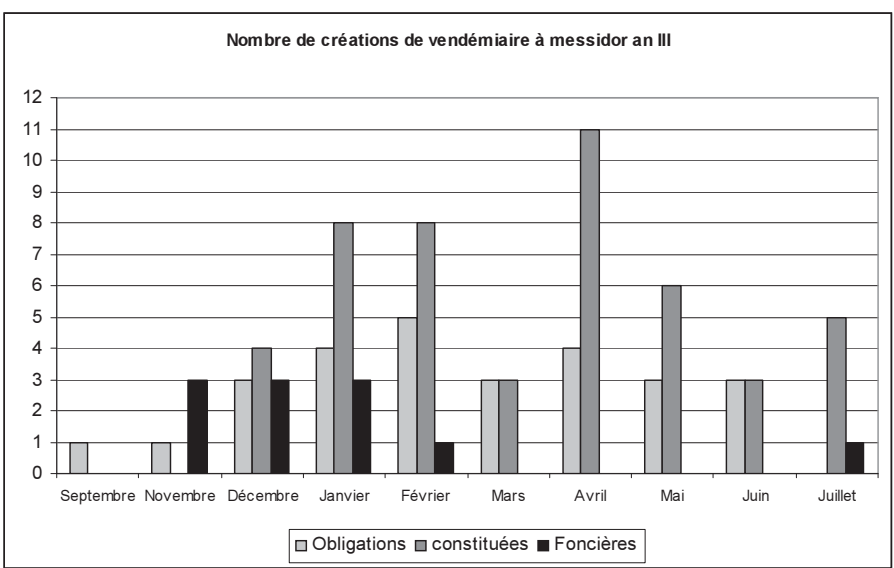

Graphique 3. Nombre de créations de rentes ou d'obligations entre vendémiaire et messidor an III.

La composition du groupe permet partiellement de comprendre le phénomène. Il s'agit de petits bourgeois d'envergure locale qui sont susceptibles d'avoir reçu en paiement ou en remboursement des assignats par poignées. À l'occasion, ils prêtent sous forme d'obligations. Il s'agit donc en principe de prêteurs avertis.

La petite bourgeoisie locale est en quelque sorte prisonnière de la situation. Que faire de la masse d'assignats que l'on détient ? La tactique qui consiste à diversifier ses placements n'est pas la pire. D'autant que si l'on dispose d'assignats on ne prend guère de risque. Dans tous les cas, le prêteur ne risque que son capital qui, de toutes façons, ne vaut pas grand-chose. Qu'a-t-il à perdre ? Il est certain que l'obligation présente plus de garantie, mais il est possible de prendre quelques précautions lors de la création d'une rente constituée. Ainsi, Louis Blondel exige que son débiteur le prévienne six mois à l'avance d'un éventuel remboursement ${ }^{36}$.

Si l'on admet que la plupart des prêts accordés durant l'an III sont libellés en assignats, ce qui est probable, on peut se demander ce qui détermine les emprunteurs à recevoir des assignats. On peut avancer au moins deux explications qui sont probablement complémentaires. La première renvoie à l'évolution des taux d'intérêt. On sait qu'en période d'argent facile le taux d'intérêt baisse. C'est le cas durant l'an III. Les rentes constituées sont en principe créées au denier 20 avant la Révolution (soit un 
taux d'intérêt de $5 \%$ ). Durant l'an III, les premières mentions inférieures à $5 \%$ apparaissent à la fin du mois de frimaire (décembre-janvier). Au-delà de cette date, environ 30 à $40 \%$ des rentes constituées sont créées à un denier supérieur à 20. Mais, le fait que le taux d'intérêt baisse ne saurait expliquer de manière satisfaisante l'existence d'un volume de prêts aussi important (63 712 livres). Les paysans, puisque pour l'essentiel ces sommes sont prêtées à des ruraux qualifiés de manouvrier, de garçons meuniers, de cultivateurs, etc., n'empruntent pas pour le plaisir de contempler des assignats, il faut qu'ils en aient l'utilité.

Dans certains cas les choses sont parfaitement claires. Le 7 nivôse l'employé note dans le registre :

«Une constitution par Marguerite Morin Ve Jacques Lépicier tutrice de ses enfants demeurant à Milly. Au profit de Louis Blondel marchand demeurant à Milly. De vingt cinq livres de rente annuelle \& perpétuelle franche des impositions païable le 21 brumaire, affectée sur une maison située à Milly ; batiments cour et jardin \& sur 75 perches d'héritage. Lad rente stipulée rachetable en un seul paiement de la somme de sept cent cinquante huit livres. Moyennant ladite somme de sept cent cinquante huit livres qui sera employé au remboursement de trente sept livres dix huit sol de rente foncière »

Un autre exemple illustre l'une des motivations possibles des débiteurs. Il concerne un très gros poisson. Le 5 frimaire, Nicolas Dupré Saint-Maur, constitue au profit de Pierre de Villerval une rente de 200 livres (au denier 25). Le scribe précise : «Laquelle somme a servi à l'emploi et paiement des domaines nationaux ». De telles précisions sont rares. Bien souvent, on ne peut que conjecturer ${ }^{37}$.

La fuite devant la monnaie emprunte parfois d'étranges détours. Un débiteur peut créer une rente constituée pour en rembourser le capital quelques semaines plus tard. On peut supposer qu'entre-temps il s'est

(37) Il faut préciser que les biens nationaux en question sont situés dans le district de Melun. Notons à ce propos que, compte tenu de l'exigüité des biens mis en vente dans la région de Milly, si ce n'est à Oncy et Soisy, le recyclage des assignats, dans le règlement des acquisitions de biens nationaux ne pouvait servir d'exutoire aux détenteurs de monnaie-papier. Laurent HERMENT, Survivant ou conquérant. : Reproduction sociale et accumulation patrimoniale chez les petits exploitants agricoles de Seine-et-Oise durant le premier XIX ${ }^{e}$ siècle (1789-1860), thèse sous la direction de Gérard Béaur, soutenue le 4 décembre 2009, p. 791 et $s q$. Voir aussi Jean-Pierre DURAND, « Utopie agraire ou force des choses ? Le cas du district d'Étampes », 89 en Essonne. Terre, propriété, et forêt, vol. 10, Comité du bicentenaire de la Révolution française dans l'Essonne, p. 40-47. 
déchargé d'une dette mais qu'il a presque simultanément subi un remboursement intempestif (sauf à considérer que c'est par amour du papier qu'il a emprunté des assignats). En définitive, on a le sentiment que chacun essaie de passer la patate chaude à son voisin.

\section{L'endettement rural. Un endettement protéiforme}

\section{Esquisse d'une typologie des opérations de crédit}

Si la rente, (sous forme foncière ou constituée), représentait un élément essentiel du système de crédit dans le monde rural que nous examinons, elle n'en constituait que l'un des éléments. Les formes et les modalités du crédit étaient infiniment variées et les actes de crédit étaient très nombreux. Pourtant, faute de source, en particulier si les parties faisaient l'économie du passage chez le notaire, il est souvent difficile, si ce n'est impossible, de les connaître. La période d'hyperinflation constitue de ce point de vue un observatoire privilégié, puisque les individus qui s'affranchissaient de dettes parfois très anciennes avaient tout intérêt à procéder à l'enregistrement des quittances que leur adressaient leurs créanciers. Ainsi, en l'an III, les sources laissent transparaître des relations de crédit interne au monde rural qui nous auraient sans doute échappé en l'absence d'inflation. L'analyse de ces relations nous permettra par ailleurs de mettre en exergue la spécificité de la vague de remboursement des rentes. En effet, si de nombreuses dettes ont été remboursées durant cette période, tous les types de dettes n'ont pas été concernés au même degré.

Nous avons longuement délaissé Anne Élisabeth Lambert. On sait que divers débiteurs lui ont remboursé plus de 20000 livres. Qu'a-t-elle fait de cette somme ? A-t-elle essayé de recycler ses assignats comme les petits bourgeois de Milly et de Maisse ? Nous n'avons relevé qu'une occurrence de ce type. Le 27 brumaire, il est fait mention d'une constitution de rente à son profit, d'un capital de 380 livres (au denier 20), par André Courtois cultivateur à Courdimanche ${ }^{38}$. C'est tout. Mais la surface sociale d'Anne Élisabeth dépasse largement le ressort du bureau. Nous savons, grâce à un enregistrement du 23 pluviôse, qu'elle a donné procuration à Nicolas Pierre Martineau de Paris « A l'effet de recevoir de qui il apartiendra la moitié des arrérages de rentes qui lui sont dus par le ci devant hôtel de ville de Paris, 
[et de] donner quittance» ${ }^{39}$. Nous avons enfin relevé une dernière mention, datée du 23 ventôse, qui permet de mesurer l'étendue de sa fortune :

«Une vente et adjudication au plus offrant et dernier enchérisseur par Anne Élisabeth Lambert veuve de Louis Ambroise Chauvin demeurante à Milly. De la coupe à faire cette année sur deux pièces de bois situées à Milly. Adjugé au cit Louis Etienne Dupré demeurant à Milly moïennant la somme de $740 £$. Devant Maricot notaire à Milly $»^{40}$

Ainsi, Anne Élisabeth et les bourgeois de Milly, si présents dans la première partie de ce travail, doivent maintenant céder la place à une foule de petits personnages qui, eux, ont cru bon de laisser des traces de leurs activités.

Il convient tout d'abord de lister les actes qui vont nous occuper maintenant. Il s'agit :

- des quittances pour règlement d'arrérages de rente,

- des quittances pour règlement de loyer,

- des quittances pour règlement de dot,

- des règlements de succession.

On peut considérer que ces actes constituent des actes de gestion normaux. Certains d'entre eux (les règlements de dots ou les règlements de succession) ont un caractère exceptionnel mais ces diverses modalités de transmission du patrimoine n'ont rien d'anormal contrairement à la vague de remboursement de rentes dont l'inflation constitue le moteur exclusif.

La présence d'autres actes est plus surprenante :

- des quittances pour le règlement d'ouvrages de maçonnerie.

- des quittances pour règlement de ventes,

- les transactions sur vente d'immeubles,

- les annulations de vente d'immeubles,

- les retraits conventionnels et l'exercice de la faculté de rachat ${ }^{41}$.

Dans ces cas on peut soupçonner que les débiteurs ont cherché à profiter de l'inflation pour se défaire de leur dette. Ces deux listes sont loin d'épuiser la variété des actes qui font explicitement ou implicitement référence à des relations de crédit interne au monde rural. Elles ont toutefois un double mérite. D'une part, elles démontrent que, loin de se résumer à

(39) Acte du 23 pluviôse devant Maricot, notaire à Milly-la-Forêt.

(40) Acte du 20 ventôse devant Maricot, notaire à Milly-la-Forêt.

(41) La liste est beaucoup plus longue. Nous avons recensé plus de 90 types d'actes différents. Le souci de synthèse doit ici sur primer le souci d'exhaustivité. 
une domination de la plèbe par la bourgeoisie par le truchement de la rente, le crédit est une composante essentielle de l'économie paysanne. D'autre part, elles permettent de rendre compte de la spécificité du mouvement de remboursement des rentes.

\section{Des actes (trop) ordinaires?}

Le caractère « normal » des règlements de loyer ou des règlements d'arrérages de rente n'a pas besoin d'être démontré. Il est par contre anormal, de trouver dans les registres fiscaux autant de quittances ou de transactions concernant les loyers (74) ou de quittances de règlement d'arrérages de rente (44). Cette profusion est évidemment l'effet de l'hyperinflation. Les locataires et les débiteurs veulent disposer d'une preuve du paiement.

Deux questions doivent retenir l'attention dans un premier temps. Les loyers sont-ils payés en temps et en heure ? Les arrérages de rente représentent-ils plus d'une année ? Sur 74 mentions de règlement de loyer, 10 font mention de retard, soit moins de $15 \%{ }^{42}$. C'est très peu. La proportion des retards dans les paiements des arrérages est beaucoup plus élevée, elle s'élève à $40 \%$. Il faut croire que les attitudes devant ces deux types de dettes sont très différentes.

On a vu plus haut que les débirentiers s'étaient délestés en masse de leurs dettes. Il semble qu'à leurs yeux, les rentes qui grevaient leur patrimoine n'avaient pas ou peu de légitimité. A contrario, la plupart des locataires règlent leur loyer sans retard. Il se peut que l'hyperinflation invite à payer ponctuellement mais les résultats obtenus incitent à penser qu'en la matière la ponctualité est la règle et non l'exception. $15 \%$ de locataires sont en retard. C'est à la fois peu et beaucoup. Cela dénote qu'en cas de difficultés, ils disposent d'une marge de manœuvre et que les dettes pour loyers impayés sont une des composantes de l'économie rurale sans en être l'un des traits saillants.

La série de quittances pour loyer présente un autre intérêt. On sait que la loi de suppression des dîmes prévoyait leur rétrocession aux propriétaires. Or de telles mentions sont très rares. Durant l'an III, dans une région où le stock de baux est composé de plusieurs centaines de baux en cours, très peu de transactions indiquent que la dîme a été rétrocédée aux

(42) Ce résultat est conforté par le calendrier des règlements. 46 règlements sont enregistrés en frimaire ou en nivôse (21 novembre 1794-19 janvier 1795). La plupart des baux sont exigibles le 11 novembre (le jour de martin). 
propriétaires. Huit enregistrements concernent ce type d'opérations. Quant aux enregistrements de baux que nous avons dépouillés ils ne mentionnent pas la dîme. On peut supposer que les propriétaires ont augmenté le prix des locations au fur et à mesure des renouvellements de baux mais, sauf exception, la suppression de la dîme ne semble pas être le déterminant de la hausse des loyers ${ }^{43}$.

Venons-en maintenant aux opérations de gestion «normales » mais « exceptionnelles ». Examinons tout d'abord les «arrangements » familiaux. Dans un pays de partage égalitaire, l'hyperinflation est susceptible de brouiller le temps et les familles. Elle peut en effet remettre en cause le fragile équilibre des transferts intergénérationnels selon plusieurs voies. On examinera deux cas de figure : les quittances pour dot et les remboursements de rente intrafamiliaux.

Durant l'an III, l'administration enregistre huit quittances pour versement de dot. Rapporté au nombre de mariages célébrés durant une année c'est assez peu. D'une part, rien n'indique que les dots sont payées avec retard. D'autre part, rien ne permet d'affirmer que les parents (ou les beaux-parents) ont profité de l'hyperinflation pour se délester massivement de ce type de dettes.

Il semble que les familles ont été très attentives aux problèmes que soulevait la prolifération d'assignats. Dans cette région les contrats de mariage sont très fréquents. De vendémiaire à thermidor an III, 67 contrats de mariage sont enregistrés au bureau de Milly-la-Forêt ${ }^{44}$. Les scribes, à la suite des notaires, et sans doute à la demande des parties, semblent noter scrupuleusement la présence des assignats dans les apports des futurs.

Au cours de l'an III, une proportion à peu près constante des apports au mariage comporte des assignats ${ }^{45}$. Tout indique que les familles anticipent ainsi les problèmes que l'existence de l'assignat pourrait poser dans un avenir plus ou moins proche. C'est d'autant plus probable que le montant moyen des apports est beaucoup plus élevé lorsqu'il est fait mention d'assignats : 2829 livres, contre 1406 livres lorsque le contrat ne mentionne pas d'assignats ${ }^{46}$.

(43) Sur l'évolution de la rente dans la région de Milly-la-Forêt, voir Laurent HERMENT, Les fruits du partage..., op. cit., p. 91 et $s q$.

(44) Dans les 13 communes qui relèvent du ressort du bureau, 522 mariages ont été célébrés entre 1792 et l'an 10, soit 52 mariages par an en moyenne.

(45) Entre 28 et $46 \%$ des apports comportent des assignats. Il n'existe aucune différence statistiquement significative entre les différentes périodes. 175000 livres.

(46) Afin de réaliser ce calcul nous avons éliminé un contrat dont les apports s'élevaient à 


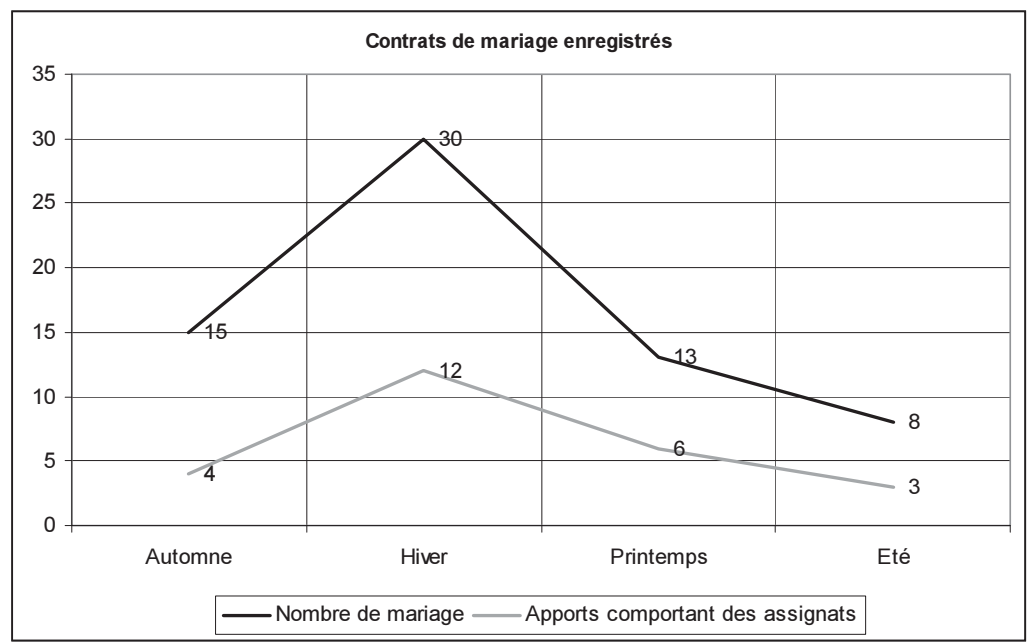

Graphique 4. Présence d'assignats dans les apports des futurs.

En ce qui concerne les règlements de succession nous disposons de deux sources d'informations : les quittances de rente et les règlements de succession. Les quittances de rente précisent parfois l'origine des obligations du débirentier. 26 quittances concernent des rentes créées à l'occasion des partages. La plupart des partages sont anciens. Ils ont fréquemment plus de 20 ans $^{47}$.

On compte par ailleurs 28 règlements de succession enregistrés durant l'an III. Le montant moyen de ces opérations s'élève à 298 livres. Leur nombre diminue régulièrement durant l'année (11 en automne, 7 en hiver, 6 au printemps et 4 en été). Il est difficile de voir dans ces chiffres une volonté de se débarrasser à très bon compte de dettes intrafamiliales. Il convient toutefois de souligner que le montant moyen des règlements crôit au cours de l'année. Il passe de 162 livres durant les six premiers mois de l'année (18 occurrences) à 543 livres durant les cinq derniers mois (10 occurrences). L'analyse des actes permet d'affirmer qu'il s'agit rarement pour un frère ou une sœur d'étrangler sa mère ou l'un des membres de sa fratrie. Parfois l'acte concerne la succession d'un oncle ; parfois il s'agit de régler les comptes avec un beau-frère ou une belle-sœur trop encombrants ; parfois c'est l'huissier qui a saisi les biens d'un défunt qui se défausse auprès des héritiers, etc. 
On le voit, il faut non seulement s'interroger sur les contours de la famille mais aussi mettre en cause le type et, accessoirement, la date des actes. Il semble que dans cette région, la règle de partage égalitaire a mis un frein aux spoliations intrafamiliales contrairement à ce qui a pu se passer dans les régions de partage inégalitaire.

\section{Les actes de gestion exceptionnels.}

La section précédente présente une vision presque idyllique des relations au sein des communautés rurales. Les actes qui retiendront ici l'attention permettront de nuancer cette impression puisqu'il s'agit d'actes qui lèsent manifestement les créanciers.

Durant l'an III, on dénombre 15 quittances pour règlement d'ouvrages de maçonnerie ${ }^{48} .11$ quittances sont passées au cours du premier semestre ; 4 sont passées durant le second semestre, lorsque l'hyperinflation devient franchement insupportable. Il n'est pas certain que les règlements aient lieu en assignats mais c'est assez probable ${ }^{49}$. Le nombre de quittances est à la fois très élevé, si l'on considère l'étroitesse de ce marché, et trop peu élevé si l'on considère le nombre de ménages qui étaient soumis à la tentation de payer en assignats. Mais il faut tenir compte de la spécificité des métiers du bâtiment. D'une part, les maçons disposaient d'un moyen de pression évident sur tous les débiteurs dont les chantiers étaient en cours. D'autre part, payer un maçon en monnaie de singe pouvait provoquer une réaction de la corporation. Si cette corporation est partiellement étrangère aux communautés rurales, elle est relativement cohérente. Durant la première moitié du $\mathrm{XIX}^{\mathrm{e}}$ on retrouve les mêmes familles de maçons : Aucordier (ou Cordier), Salar (ou Salard ou Salars), Couderc (ou Coudair), Fort (ou Faure). Elles sont souvent originaires du Limousin.

Les dernières opérations qui retiendront l'attention concernent les ventes d'immeubles. On distinguera trois types d'opérations : l'exercice de la faculté de réméré et les retraits, les règlements de ventes d'immeubles ${ }^{50}$, les transactions sur ventes d'immeubles et les annulations de vente.

L'exercice de la faculté de réméré n'est pas une pratique très fréquente durant l'an III. On compte 16 opérations de ce type ${ }^{51}$. Le montant moyen

(48) Ces quinze contrats concernent treize créanciers. Dans deux cas il s'agit de travaux de couverture exécutés sans doute par des couvreurs en chaume.

(49) Les marchés en cause sont rarement datés. Lorsque c'est le cas, ils ont été formés entre

1792 et 1794 . Ce délai de un à trois ans est cohérent avec ce que l'on sait de ce type d'activité.

(50) Il peut s'agir de reliquat sur vente d'immeubles.

(51) Les scribes intitulent ces opérations « retrait conventionnels » ou « réméré ». Il y a aussi une opération de «transaction sur réméré ». Le réméré, parfois nommé « pacte de rachat », est un acte 
de ces opérations s'élève à 327 livres. Ces transactions mettent aux prises des laboureurs, des manouvriers, des vignerons, des cultivateurs ou leurs ayants droit (leur veuve et leurs enfants). La bourgeoisie est totalement absente à l'exception du citoyen Fautray, officier de santé, domicilié à Milly. Le faible nombre d'opérations indique que la vente avec faculté de réméré est une pratique peu répandue dans cette région. Mais dans ces cas, la volonté de profiter de la dévalorisation de l'assignat est évidente. Les débiteurs exercent leur faculté de réméré tardivement. Neuf actes sur treize sont postérieurs au premier floréal, à un moment où l'assignat ne vaut presque plus rien. Les créanciers perdent jusqu'à $95 \%$ du montant de la vente.

Les règlements de vente d'immeubles et les reliquats de dettes sur ventes d'immeubles constituent le second type d'opérations. Il s'agit là encore d'opérations essentiellement internes au monde rural. Parfois, elles impliquent quelques bourgeois comme Jacques Pichard, libraire, domicilié Quai Voltaire à Paris, époux de Jeanne Margueritte Pélagie Fortemps. Les montants en cause sont très importants : plus de 1900 livres en moyenne. L'échelonnement dans le temps des treize actes souligne la volonté de la part des débiteurs de profiter pleinement de la dégringolade de l'assignat à partir de germinal (Tableau 1).

Tableau 1. Règlements de vente d'immeubles et reliquats de dette sur ventes d'immeubles ${ }^{\mathrm{a}}$.

\begin{tabular}{|l|l|l|}
\hline Date de Remboursement & Nombre d'opérations & Montant moyen (en Livres) \\
\hline Brumaire & 1 & 60 \\
\hline Pluviôse & 1 & 184 \\
\hline Germinal & 4 & 2734 \\
\hline Floréal & 3 & 967 \\
\hline Messidor & 4 & 2885 \\
\hline
\end{tabular}

a. Il s'agit de montants indicatifs. Certaines créances sont plus importantes et ont été partiellement remboursées auparavant.

Dans certains cas, ces opérations concernent les membres d'une même famille. Ce constat contredit la vision presque idéale des rapports familiaux qui a été évoquée plus haut. Mais, même en supposant que toutes les quittances pour règlement de ventes d'immeubles constituent des quittances intrafamiliales, ce qui est loin d'être le cas, ces comportements

par lequel le vendeur se réserve le droit de reprendre la chose vendue en contrepartie de la restitution du prix. 
restent très minoritaires. Or, répétons-le, dans la plupart des cas il ne s'agit pas de créances intrafamiliales.

Si les comportements évoqués précédemment sont marginaux, la tentation devenait trop forte pour un nombre croissant de débiteurs. Pour se protéger les créanciers devaient gagner du temps ou, du moins, parvenir à faire croire qu'ils étaient en mesure d'en gagner. C'est ainsi qu'à partir du 20 germinal un nouveau type d'actes apparait dans le registre : les transactions (ou les accords) sur vente d'immeubles. L'enregistrement du 24 germinal est particulièrement clair :

«Un accord entre Louis Charles Charlot perruquier demeurant à Milly et Julienne Nardon sa femme. Et Claude Nardon demeurant à Milly. Par lequel ledit Charlot renonce à demander aucune chose relativement à la vente qu'il a faite aud Nardon en l'année 1785, d'un jardin sis à Milly moïennant soixante quinze livres. Moïennant que ledit Nardon a païe en sus du prix la somme de vingt cinq livres. Par acte sous seing privé à Milly le jourdhuy [24 germinal an III] »

On peut imaginer que, devant le mauvais vouloir du créancier, le débiteur s'est résolu à payer un supplément de prix. Mais que gagne le créancier s'il est payé en assignats ? Rien ou presque. Il faut imaginer que les débiteurs ont payé le supplément en bons deniers métalliques ou que les débiteurs offrent une compensation ou un dédommagement en nature à leur créancier, ce qu'indiquent certains actes.

Au total, on ne compte que 16 opérations de ce type. Soit ce type de créances était très rare parce que les débiteurs se libéraient très vite de leurs dettes comme le suggère Gérard Béaur ${ }^{52}$, soit les débiteurs n'ont pas voulu (ou n'ont pas osé) profiter de la dévalorisation de l'assignat. Ces deux hypothèses ne sont pas exclusives l'une de l'autre.

Le comportement variable des débiteurs permet de mieux comprendre la déconfiture d'Anne Élisabeth Lambert. Reprenons pour conclure le dossier Lambert. Comme nous l'avons vu, Anne Élisabeth est représentative de la classe des rentiers bourgeois dont l'assise sociale se limite parfois à une microrégion ou, au contraire, en dépasse les frontières. Cette classe sociale est la première victime du mouvement de remboursements. Mais,

(52) Gérard BÉAUR, « Des sols contre de l'argent. L'argent dans les transactions foncières au XVIII ${ }^{\mathrm{e}}$ siècle », dans Philippe MINARD et Denis WORONOFF (dir.) L'argent des campagnes. Échanges, monnaie, crédit dans la France rurale d'Ancien Régime, Journée d'étude tenue à Bercy (2000), Paris, publication du CHEFF, 2003, p. 171-183. 
comme ses pairs, Anne Élisabeth ne subit pas d'autres dommages trop importants. La position de ces rentiers permet d'avancer deux conclusions. La première concerne l'existence d'un double circuit de la rente : un premier circuit, que contrôlent les rentiers, et un second circuit, qui est interne à la société rurale et qui est le produit de la règle de partage égalitaire et des mécanismes de circulation de la terre. Ces deux circuits s'effondrent simultanément. La seconde conclusion renvoie au système de crédit interne à la société rurale qui ne se limite pas à la rente ou aux autres types de crédits formels. Il concerne pratiquement toutes les formes de l'activité économique (nous n'avons envisagé ici que quelques-unes des modalités). Il n'émerge que partiellement à la faveur de l'hyperinflation. Mais il permet de mieux apprécier l'importance de la vague de remboursement des rentes.

La paysannerie semble avoir établi une distinction claire entre ce qui lui paraissait légitime et ce qui constituait à ses yeux une anomalie. L'existence des rentes heurtait sans aucun doute la volonté de la paysannerie de se libérer d'un mode de domination qui n'avait plus de justification à ses yeux. La rente foncière et les rentes constituées auraient eu en quelque sorte un destin semblable à celui des droits féodaux et des dîmes. Rafe Baufarb note que « bien loin de constituer le concept fondateur et rationnel que les révolutionnaires désiraient, la propriété généra une lutte interne entre propriétaires rivaux $»^{53}$. De ce point de vue, dans leur lutte contre la bourgeoisie, les paysans de la région de Milly-la-Forêt sortent grands vainqueurs.

Les autres types de dettes étaient beaucoup moins exposés, sans doute parce que leur existence était justifiée aux yeux des populations rurales. Les retards de paiement dans les loyers sont rares. Il est remarquable de constater que les rentiers du sol qui possèdent un patrimoine locatif important ne sont pas touchés. Ils disposent de surcroît d'une arme de dissuasion redoutable : le non-renouvellement du bail. Quant aux règlements de dettes liées à l'acquisition des terres ils sont exceptionnels.

Enfin, l'hyperinflation n'a pas constitué un ferment de désagrégation des relations familiales. Dans les régions de partage égalitaire il faut y regarder à deux fois avant de léser son frère ou sa sœur en profitant de la dévalorisation des assignats. Cela entraîne presque automatiquement la remise en cause du partage savamment concocté par les parents. De ce

(53) Rafe BlAUFARB, «Propriété, politique et délimitation des groupes sociaux : le débat sur les rentes foncières, 1789-1811 », AHRF, n 359, janvier-mars 2010, p. 119-140, p. 120-121. 
point de vue il semble que le système de partage ait constitué un garde-fou à défaut d'être une assurance tous risques.

En l'état, cet ensemble de conclusions n'est toutefois pas totalement satisfaisant pour deux raisons. En premier lieu, les familles, comme nous l'avons vu, n'ont pas été épargnées. L'explication de ce phénomène est en soi assez simple. Une fois le mouvement de remboursement enclenché, l'effet domino semble avoir joué à plein : un remboursement entraînant une cascade de remboursements, y compris au sein des familles. La rente foncière qui constituait un mécanisme commode de fluidification des transferts intergénérationnels a dès lors perdu une grande partie de son rôle d'autant que les familles disposaient de moyens alternatifs pour assurer l'égalité entre héritiers. Une étude sur les modalités de partage au sein des fratries durant la première moitié du $\mathrm{XIX}^{\mathrm{e}}$ siècle permettrait sans doute de répondre avec précision aux interrogations que soulève cette remarque $^{54}$. En second lieu, si l'on admet que la rente foncière avait perdu une grande partie de sa légitimité, il faut bien admettre que, jusqu'à la veille de la Révolution, elle conserve ses attraits comme moyen d'accès à la terre ou comme moyen de circulation de la terre. Faut-il voir dans sa liquidation un dommage collatéral de Révolution ? Cette remise en cause ne constituerait en définitive que l'un des aspects de la Révolution économique dans les campagnes. La vague de remboursements, en purifiant la domination bourgeoise de ses formes désuètes et en la réduisant à un rapport de propriétaire à locataire, aurait alors agi à la fois comme une catharsis et comme un cordial.

\section{Annexe I}

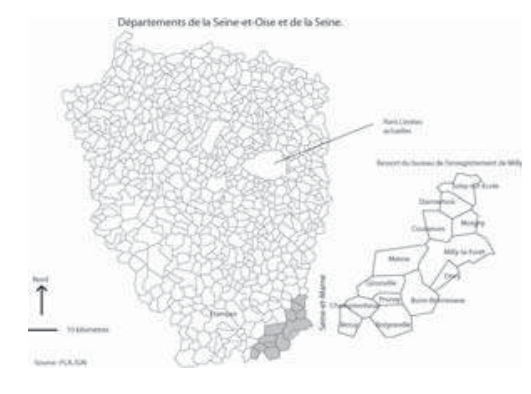

(54) Sur la structure de l'endettement de la paysannerie et son évolution au cours de la première moitié du XIX ${ }^{\mathrm{e}}$ siècle dans la région de Milly-la-Forêt, voir Laurent HERMENT, Les fruits du partage..., op. cit., p. 309-317. 


\section{Annexe II}

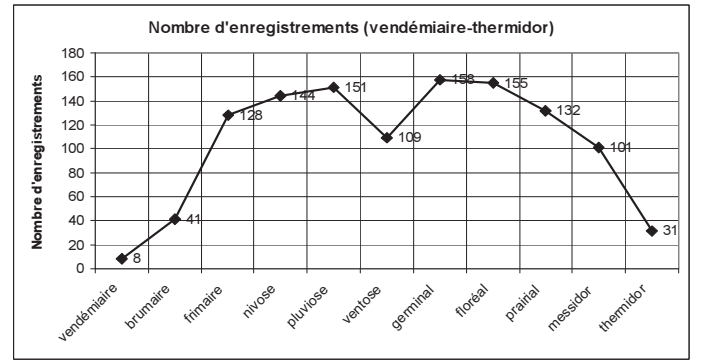

Graphique 5. Mouvement de remboursements de rentes à Milly-la-Forêt.

Laurent HERMENT

CNRS-CHR, Paris, UMR 8558

10 boulevard de Stalingrad,

92320 Châtillon.

herment@hotmail.com 